\title{
Nachlese zu: Der Fenstersturz und das Bundesgericht
}

\author{
Die SÄZ-Leser und -Leserinnen erinnern sich: Gemäss Bundesgericht hätte das \\ betreffende Universitätsspital eine Sitzwache stellen sollen; diese hätte für \\ diesen Patienten für eine Woche ja nur rund Fr. 1680.- gekostet. Das Bundes- \\ gericht hat dabei die «number needed to treat»-Frage übersehen [1]. Zwei so- \\ eben erschienene Urteilsbesprechungen von Rechtsprofessoren bestätigen \\ unsere letztjährige kritische Analyse des Bundesgerichtsentscheides.
}

Hanspeter Kuhn, Fürsprecher, stv. Generalsekretär
Heinz Hausheer, alt Bundesrichter und emeritierter Professor an der Universität Bern kommentiert: «Wollte man mit der vom Bundesgericht verlangten Gleichsetzung von genereller Vorhersehbarkeit und objektiver Vorwerfbarkeit wirklich Ernst machen, hätte dies im Gesundheitswesen eine Kostenexplosion unabsehbaren Ausmasses zur Folge. Das lässt doch vermuten, dass der überaus strenge Massstab, den das Bundesgericht in der vorliegenden Streitsache unter Hintanstellung der bei der Willkürkognition ansonsten befolgten Zurückhaltung - an die Beurteilung des ärztlichen Sorgfalt angelegt hat, ein durch die Besonderheit des Einzelfalls begründeter «Ausreisser» ist. Als allgemeine Regel kann und darf der Entscheid nicht Schule machen.» [2]

Hansjörg Seiler, Bundesrichter am EVG und Titularprofessor an der Universität Luzern, ergänzt in seinem lesenswerten Beitrag «Wie viel Sicherheit wollen wir? Sicherheitsmassnahmen zwischen Kostenwirksamkeit und Recht»: «Die Rechtsordnung hat noch nie verlangt, dass sämtliche Risiken eliminiert werden. Verlangt wird die Einhaltung der zumutbaren Sorgfalt. Es muss ein angemessenes Verhältnis zwischen dem Sicherheitsaufwand und dem erreichten Sicherheitsgewinn angestrebt werden.» Sein Kommentar zum Fenstersturzfall lautet: «Das Bundesgericht hat den [...] Denkfehler began- gen: Es hat die Kosten der Sicherheitsmassnahme nicht in Relation gesetzt zu den dadurch vermiedenen Risiken, sondern lediglich zum nun eingetretenen Schaden, ohne die Wahrscheinlichkeit des Ereignisses zu berücksichtigen.» Seiler schliesst: «Würde man bezüglich aller denkbarer Risiken Sicherheitsmassnahmen vorschreiben, die ein derart schlechtes KostenWirksamkeits-Verhältnis haben, dann würde nicht nur das Gesundheitswesen, sondern die gesamt Volkswirtschaft von heute auf morgen kollabieren.» [3]

Der Transparenz halber ist zu erwähnen, dass dieser Autor damals am Entscheid des kantonalen Verwaltungsgerichts mitgewirkt hatte, der dem Bundesgericht so missfiel. Das ändert nichts daran, dass auch seine Kritik - wie die des am Fall nicht beteiligten Prof. Hausheer - zutrifft.

\section{Literatur}

1 Kuhn HP. Der Fenstersturz und das Bundesgericht. Schweiz Ärztezeitung. 2006;87(23):1015-7.

2 Hausheer H, Jaun M. Die haftpflicht- und privatversicherungsrechtliche Rechtsprechung des Bundesgerichts in den Jahren 2002-2005. Zeitschrift des bernischen Juristenvereins ZBJV. 2007; 143:103ff, S. 123.

3 Seiler H. Wie viel Sicherheit wollen wir? Zeitschrift des bernischen Juristenvereins ZBJV. 2007; 143:140ff, S. 150. 